\title{
The Ultimate Haptic Device: First Step
}

\author{
${\text { Guillaume Millet* } \quad \text { Sinan Haliyo }{ }^{\dagger} \quad \text { Stéphane Régnier }}^{\ddagger} \quad$ Vincent Hayward $^{\S}$ \\ UPMC Univ Paris 06, UMR 7222, \\ Institut des Systèmes Intelligents et de Robotique, F-75005, Paris, France
}

\section{Abstract}

We describe a single-axis haptic interface which is based on a dualstage actuator technique and which is aimed at achieving perfect transparency to a human user. The paper shows how all parasitic forces arising from inertia and friction can be brought below human detection thresholds, yet, the system is able to output significant torque. It has a stage with a large motor coupled to a distal stage with a smaller motor via a viscous coupler based on the principle of eddy current induction. The paper also describes its control principle and preliminary results.
\end{abstract}

Index Terms: H.5.2 [Information Interfaces and Presentation]: User Interface-Haptic I/O; H.5.1 [Information Interfaces and Presentation]: Multimedia Information Systems-Articial, augmented, and virtual realities; H.1.2 [Information Systems]: User/Machine Systems-Human information processing

\section{INTRODUCTION}

The ultimate haptic device has no mass and has infinite bandwidth. In an effort to approach this ideal, it is worth considering what bounds performance above, that is, what is the maximum performance that can possibly be achieved, and what bounds it under, that is, how good performance should be. In the area of force feedback devices, the actuator performance is what bounds the performance above. With a given actuator, no manner how well transmissions and linkages, if any, are designed, these elements can only degrade key aspects of performance such as the dynamic range (ratio of the largest to the smallest specifiable force), the stiffness of the connection between the prime mover and the manipulandum, or the end-point inertia.

In this paper, we describe an approach to the design and construction of a single-axis haptic interface prototype that can cover a significant portion of the range of human sensorimotor performance. Perhaps, the most relevant aspect of human performance with respect to haptic device design, is the smallest humandetectable force. In an ideal device, the smallest detectable force should match the smallest force that can be commanded by the device and the largest force should match the normal operation of the human hand.

While actuator saturation (long and short term) is clearly the factor that determines the upper limit of the range, the determination of the lower limit merits some discussion. When a manipulandum interacts with a hand, it is subject to a number of forces (to simplify the discussion, for now no distinction is made between forces and torques). If we can neglect the effect of internal elastic forces, that is, if the device operates below its first resonant mode, these forces are: the actuator force, typically a Lorentz force developed in the motor windings; the forces due to viscous and friction losses

\footnotetext{
*e-mail: millet@isir.fr

†e-mail: sinan.haliyo@upmc.fr

†e-mail: stephane.regnier@upmc.fr

$\S$ e-mail: vincent.hayward@isir.fr
}

(if the device has sliding surfaces), plus those due to losses arising from induced currents and which also oppose motion; inertial forces to which each moving part is subjected; and the force applied by the hand which includes at least an inertial component, a viscous component, and an elastic component, all due to the movement of tissues. According to Newton's second law, all these forces must balance.

We now turn our attention to the problem of the design of a transducer able to command a known force at the interface between a manipulandum and the relatively soft load represented by a twofinger grip. We also desire that this force be commanded, not only at DC, but over a wide frequency range. It follows from the previous discussion that parasitic forces resulting from inertia and losses should be negligible. But how small is negligible?

We can look at this question from one of two possible, hopefully equivalent, viewpoints. Seen from the view point of the device, we could strive to engineer it so that the parasitic forces are smaller that the smallest human-detectable force under all desired operating conditions. Seen from the viewpoint of the hand, we could desire the device to have a mechanical impedance (ratio of force over displacement and derivative) that is significantly smaller than that of the fingertips since then, tissue deformations would not be the result of parasitic forces but from only commanded forces. We will examine some plausible numerical values in Section 3. It will soon become clear that a single standard electric motor, the actuator of choice when building impedance-based haptic devices, will not meet the desired objective.

The rest of this paper describes a two-actuator system depicted in Fig. 1 which is based on the principle of coupling a large actuator to a small one via a viscous coupler such that, seen from the manipulandum, the system's apparent mechanical impedance and risetime is that of the small motor but the largest force it can deliver is that of the large motor. If we can design a system which has these properties, then extremely high-performance can be achieved.

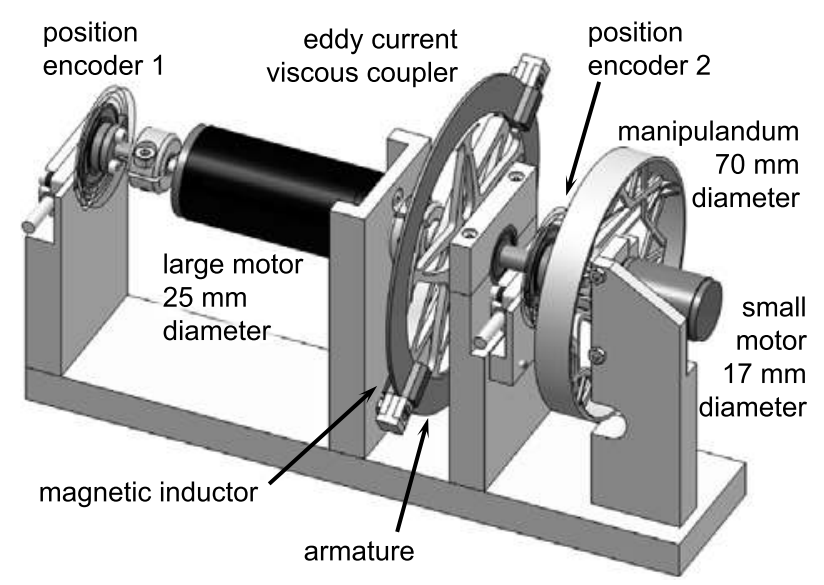

Figure 1: Computer-aided design representation of the prototype device showing the main components (Catia, Dassault Systèmes). 


\section{Related Approaches and a New One}

Dual-stage robotic devices go a long way back. There are several reasons for wanting to have more than one actuator per joint. Perhaps one of the oldest motivations is the desire to modulate the intrinsic dynamics of an actuated joint in a effort to imitate the antagonist action of skeletal muscles. References $[27,8,26]$ describe examples of this approach to modulate elasticity with electric motors, and reference [2] discusses the modulation of viscosity with hydraulic motors. Recently these ideas have been developed further in a effort to make robots that can interact with people safely and which are surveyed in [1].

It is customary to use the word 'series' and 'parallel' to discuss the coupling options between mechanical elements. Unfortunately, this terminology can be confusing. In electrical circuits, when two elements are 'in-series' they share the same current. Confusion arises because there are two possible analogies between the mechanical and the electrical domains [16], the same mechanical elements could be 'in-series' or 'in-parallel' according to a choice. To prevent confusion, we discuss the methods of coupling between motors and loads, and avoid using the terms 'series' and 'parallel'.

In the symbolism of Fig. 2, a rectangular box represents an inertia, two parallel lines represent a controllable source of force, typically a Lorentz force, a coil represents a spring, a piston represents a damper, a small circle represents the load, and a mechanical connection is a connection (common velocity). Referring to this figure, case $\mathbf{a}$ is an important one because it models many actual haptic devices. In effect, a preferred method of construction of these devices calls for grounding the actuators, therefore, there is at least an elastic element between the motor and the handle. If elasticity is built-in by design, then we have so-called "series elastic actuators" [20, 15], but for haptics we would rather try to make the spring as stiff as possible to raise the system natural frequency [11]. Configuration $\mathbf{b}$ corresponds to the standard, variously called coarse-fine or minimacro manipulator design $[22,23,21,14,12]$. Here a large motor 'carries' a smaller one, but both share the same load since they are on the same load path. Such a scheme has been advocated for haptic devices $[25,30]$. With proper control this configuration can reduce the apparent inertia of the whole system but the small motor must be able to bear the whole brunt of the load. This naturally leads to the arrangement $\mathbf{C}$ described in reference [17] which does not have this limitation and which also can be applied to making human-friendly robots [32]. The large motor supplies the largest portion of the force and the small motor "fills-in" during transients. With proper control, the user is exposed to the inertia of the small motor but not that of the large. A variant design is proposed in reference [5] where the large motor is replaced by a brake.

The haptic designer can use all three types of forces: elastic, dissipative, and inertial. From this view point, configuration $\mathbf{d}$ is an option. In fact, it is by inertial coupling (and other tricks) that portable phones and gaming pads give 'force feedback'. So why not consider option $\mathbf{e}$ to achieve an effect similar to that of arrangement C? It is in principle possible but probably too complex to be practical. A first problem is the need for a slip-ring electrical connection, or other mechanisms accomplishing a similar function. A second problem is the need to include an additional control mechanism able to bring the "fly-wheel" velocity to zero on average.

This leaves us with dissipative forces to achieve desirable couplings. The counterpart of case $\mathbf{a}$, case $\mathbf{f}$, is described in reference [4] for robotic applications. At first sight, it is not very appealing for haptics since, say with an elastic load like a hand, this coupling will introduce an attenuation of $6 \mathrm{~dB} /$ octave on top of the $12 \mathrm{~dB}$ /octave attenuation due to the motor inertia. Although we could think of option $\mathbf{g}$ to 'take up' the response in the highfrequency, similarly to $\mathbf{b}$, such configuration would have the same problems. Option $\mathbf{h}$ is worth mentioning. It has been shown to be able to generate distorsion-free high-frequency haptic information a

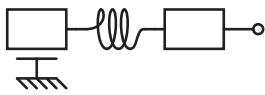

b
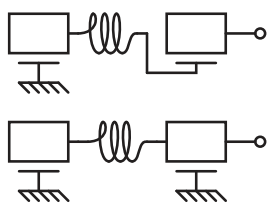

d

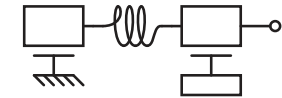

$\mathbf{f}$

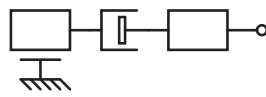

g

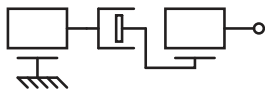

h

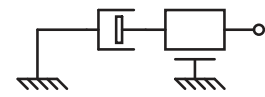

i

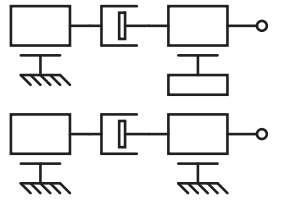

Figure 2: Several ways one or two motors can been coupled to a load. See text for discussion. Combination $\mathbf{j}$ is the combination adopted for the 'ultimate haptic device' described in this article.

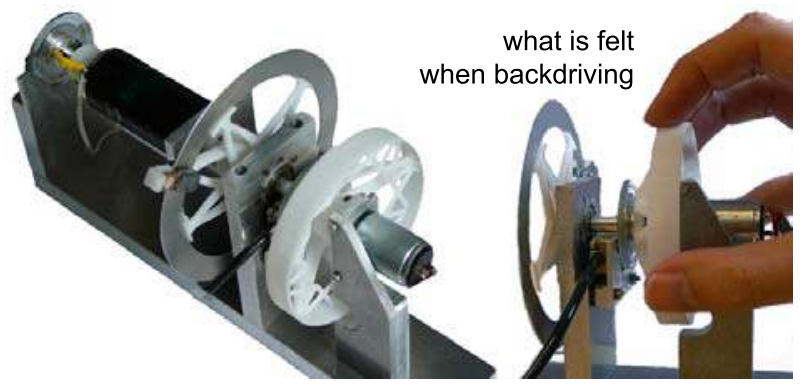

Figure 3: Prototype realized according to strategy j. Under proper control, the perceived inertia is that of a thin aluminum annulus plus a light-weight handle. Friction is negligible since it is a non-contact transmission relying on eddy current induction. This is illustrated in the right panel which shows only the moving parts that are felt.

up to $100 \mathrm{~Hz}$ [3]. We could propose the arrangement $\mathbf{i}$ but it would suffer from the same difficulties as $\mathbf{e}$. This leaves us with design $\mathbf{j}$ which is further discussed in Section 4. Figure 3 shows our first realization where the coupler is a non-contact eddy-current clutch.

\section{Performance Estimates}

How good is good? The parasitic forces in a haptic device can, again, be put into three categories: inertial forces, dissipative forces, and elastic forces. For the later, we may simply consider that the effect of elasticity in a transmission is the occurrence of structural dynamics (resonances and anti-resonances) and hence can be quantified by the lowest natural frequency, $F_{0}$, that limits the range of operation. The other forces can be simply quantified by their greatest magnitude over the range of operation [11].

\subsection{Time-Domain Performance of Fingertip Detection}

The sought-after number depends on the task. One approach is to consider the human ability to discriminate short temporal events. According to reference [18], we can situate this number at $30 \mathrm{~ms}$. On the other hand, vibrotactile studies, typically performed by measuring the displacement of an object in contact with the skin [28], indicate that fingertip displacements of the order of $1 \mu \mathrm{m}$ can be detected at $1 \mathrm{kHz}$, giving a number closer to $1 \mathrm{~ms}$. Such a difference is to be expected since discrimination is a harder task than detection. In terms of a performance figure, we would desire a system risetime of $1 \mathrm{~ms}$ in the small signal range. 


\subsection{Smallest Human-Detectable Force at the Fingertip}

There has been work on the discrimination of forces at the fingertip. One of the most relevant to our present purpose is described in reference [31]. Unfortunately, the present authors could find only one study where subjects were tested with very low forces [7], at the detection threshold, either statically or dynamically (testing with von Frey filaments wouldn't not be appropriate here), probably due to the limitations of current electromechanical stimulus generation methods. Nevertheless, we can arrive at a number indirectly. There are now good estimates of the tangential stiffness of a fingertip [19], suggesting the figure of $0.210^{3} \mathrm{~N} \cdot \mathrm{m}^{-1}$. As for the smallest detectable skin displacement, unfortunately the authors could not find a direct study either. Yet, from vibrotactile studies the smallest detectable displacement is very small, of the order of $10^{-7} \mathrm{~m}$ ! [28]. But it is implausible that at several hundred $\mathrm{Hz}$, the interaction force be entirely attributed to elastic forces in the fingertip-there are also viscous and inertial forces at this frequency. The smallest detectable force would be extremely small, viz. $10^{-4}$ N. Statically, the skin can also detect very small displacements, but accounting for the discussions in references [13, 24], we could revise the estimate to $10^{-5} \mathrm{~m}$, putting the smallest detectable force at $10^{-2} \mathrm{~N}$ in the low frequencies. As a sanity check, consider that the mass of a US cent coin is $2.5 \mathrm{~g}$. When it is held between two fingers it statically loads the skin by about $10^{-2} \mathrm{~N}$ on each. This load seems to be at about static threshold. In summary, an ultimate device should specify forces at the fingertip within $10^{-2} \mathrm{~N}$ statically and within $10^{-3} \mathrm{~N}$ dynamically.

\subsection{Hand Motor Capabilities}

Given the vast range of human motor capabilities, perhaps the most reasonable manner to estimate the largest acceleration and velocity that can be expected is to consider a specific motor task such as reaching a target. The key reference is [9] where it is found that people tend to spend $0.2 \mathrm{~s}$ to reach a target separated by $5 \mathrm{~cm}$ from the initial position. At the scale considered, assuming a parabolic trajectory profile, from these numbers we can deduce that the finger acceleration could be roughly $2 \mathrm{~m} \cdot \mathrm{s}^{-2}$ and that the velocity could reach $0.2 \mathrm{~m} \cdot \mathrm{s}^{-1}$. Accelerations and velocities are certainly much higher in non-informational and in ballistic tasks, but these need not be considered for a haptic interface.

\subsection{Target Performance Figures}

By combining the numbers found in the previous sections we are now in a position to specify the target performance figures for a haptic interface that can operate at the limits of human performance. These numbers are arrived at by considering that when the hand actively interacts with the handle, parasitic forces should be at threshold, thus not interfering with the Lorentz force produced by the motors. The results are collected in Table 1.

Table 1: Target performance figures and their angular equivalents are given for the case of a $70 \mathrm{~mm}$ handle.

\begin{tabular}{lcccc} 
Quantity & Unit & Value & Angular Unit & Value \\
\hline Mass $^{\mathrm{a}}$ & $\mathrm{kg}$ & $0.510^{-2}$ & $\mathrm{~kg} \cdot \mathrm{m} 2$ & $61.310^{-7}$ \\
Viscosity $^{\mathrm{b}}$ & $\mathrm{N} \cdot \mathrm{s} \cdot \mathrm{m}^{-1}$ & $0.510^{-1}$ & $\mathrm{~N} \cdot \mathrm{m} \cdot \mathrm{s} \cdot \mathrm{rad}^{-1}$ & $61.310^{-6}$ \\
Friction $^{\mathrm{c}}$ & $\mathrm{N}$ & $1.010^{-3}$ & $\mathrm{~N} \cdot \mathrm{m}$ & $35.010^{-6}$ \\
Risetime $^{\mathrm{d}}$ & $\mathrm{S}$ & $1.010^{-3}$ & & \\
Max force & $\mathrm{N}$ & 5.0 & $\mathrm{~N} \cdot \mathrm{m}$ & $17.510^{-2}$ \\
a obtained from largest acceleration and lowest statically detectable force. $^{\mathrm{e}}$ & $\mathrm{b}_{\text {obtained from largest velocity and lowest statically detectable force. }}$ \\
c obtained from lowest dynamically detectable force (reversal transients). \\
d obtained from vibrotactile performance. \\
e obtained from common sense.
\end{tabular}

It is worth noting that the dynamic range from the lowest to the highest specifiable force turns out to cover four orders of magnitude and that the achievable dynamic range of a high-quality DC motor is only about two orders of magnitude. This observation vindicates the need for at least a two-stage design.

\section{DESIGN}

\subsection{Rationale}

Dual stage arrangements are common in electronic amplifier design (such as the "current dumping audio amplifier" of the QUAD company [29] or the classic Doherty scheme [6]) where a powerful amplifier supporting the brunt of the load is coupled to a high-quality fast-stage to fill-in the signal that the primary stage cannot supply during transients. Here we have a similar approach.

The spring coupling is an interesting coupling option, but springs have the property of storing energy. This is an advantage in applications where energy conservation is desirable, such as in a walking machine, but in haptics, energy storage is inconvenient, even hazardous. Humans interacts with devices typically at rather low velocities. For an elastic coupling to output a large torque, it must be wound up. If the output torque needs to be brought to zero abruptly, for instance when breaking contact with a virtual wall, the feedback system must be able to unwind the coupling at high speed since displacement is two integrals away from the output torque. Moreover, when letting go of a handle during interaction, the elastic energy would be uncontrollably converted to kinetic energy almost instantaneously, causing a hazard, since the output stage must be light and weak, hence unable to control movement.

A viscous coupling, in contrast, does not have these problems, or at least to a lesser extent. To produce torque, the large stage must spin, also storing energy in kinetic form. But this energy cannot transfer at the output transiently since the coupling is dissipative by construction. It will tend to dissipate energy in itself rather than in the user. Moreover, in contrast to the elastic coupling case, the output torque is only one integral away from the output torque. Hence, the basic servo mechanism that governs the large stage is a velocity servo, which is much easier to realize than a position servo (system has at least one fewer order). As an added benefit, the large stage can react more quickly than in the case of an elastic coupling, relying less on the small stage to produce an accurate output.

There are several other advantages of the viscous coupling over the elastic coupling. One of them is accuracy. Dual stage designs produce an accurate output force because, in essence, they are force-feedback systems. The feedback control system in an elastically coupled devices essentially attempts to regulate a spring deflection in a resonant system. So the design is complex, and if the spring is not of exacting quality, there will be hysteresis and distributed high-frequency modes. The viscous coupling in the present proposal is based on eddy current induction. This coupling technique is non-contact and is for all practical purposes perfectly linear (within many orders of magnitude) provided that the velocity is small enough. In haptics, the velocities of interest are much below the critical velocity at which an eddy current clutch becomes nonlinear [10]. Provided that the relative velocities of the two shafts can be measured accurately, the viscous coupler is nothing but a very high quality non-contact force sensor.

\subsection{Control System}

For the time being and for experimentation purposes, we implemented a simple scheme. A simplified model of the electromechanical behavior of a device, as in Fig. 1 and Fig. $2 \mathrm{j}$, is represented in Fig. 4, dashed line box. We have two electric motors driven in current mode and coupled by a viscous clutch $b$. The coupling produces an output torque, $\tau_{\text {out }}$, which is (exactly) proportional to the speed difference of the two shafts, give or take the inertia, $J_{2}$, of the distal stage and friction, $\tau_{2 \text { fric }}$. The control system 


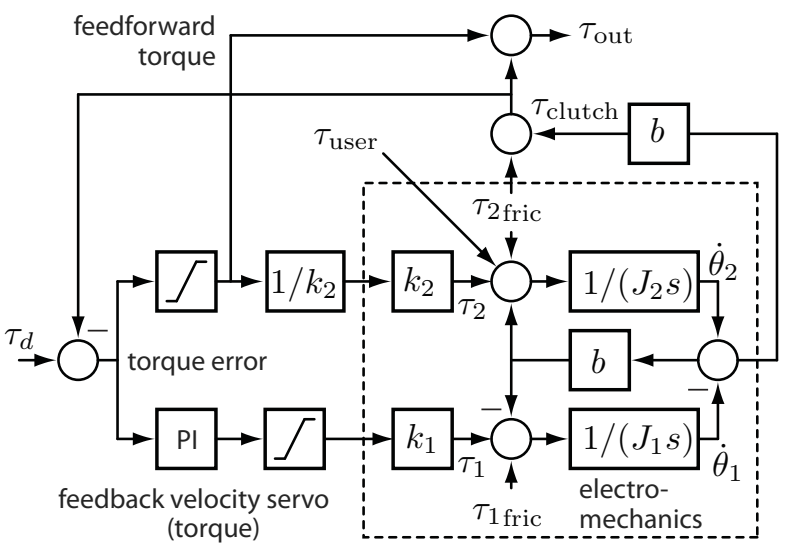

Figure 4: Control block diagram.

has a feedback path to regulate the differential velocity of the two shafts. If this regulation is sufficiently good, then the dynamics of the large motor completely disappears from the user's experience. What is felt is only the distal moving parts. Since people move rather slowly by electromechanical standards, achieving this regulation is not particularly difficult. For an initial design, a standard PI regulator gave excellent results since the system has only one stable pole and the main disturbance is the shaft friction.

Interestingly, unlike other haptic interface designs, the torque applied by the user, $\tau_{\text {user }}$, is not a disturbance. Its effect is to cause the movement of a very light handle, which, in turn, appears as a setpoint to the control of the large motor. The control is therefore robust by construction. This scheme relies on knowing the coefficient $b$ precisely. In the case of eddy current coupling, this coefficient remains constant over a wide range of velocities and can be known very precisely. The risetime is of course limited by the time constant of the velocity servo of the large motor. To compensate, the torque error is simply sent to the distal stage motor in open-loop, which also requires that the motor torque constant, $k_{2}$, be known. As a result, the control is not only intrinsically robust but unconditionally stable for any type of load. The risetime of the whole system is that of the distal stage. The control system was implemented using a PC running the RTAI real-time Linux kernel and the sampling rate was set at $5 \mathrm{kHz}$.

Although we have not yet checked this result rigorously, because of the channel separation, it is expected that our design will remain robust when included in the loop of a virtual environment or of a teleoperation system. The system also lends itself nicely to future improvements including more advanced optimal control designs.

\subsection{Prototype Description}

In our dual-stage device, structural dynamics will have an effect only in the distal stage which must be made light and stiff. The main design challenge is the construction of the eddy-current clutch briefly described next. To construct a first prototype, we simply employed two motors that were unused in the lab. They turned out to be a conventional coreless DC motor (model RE-25 graphite brushes, Maxon Motors ag, Sachseln, Switzerland) for the proximal stage and a small $17 \mathrm{~mm}$ motor (model 1724, Faulhaber $\mathrm{GmbH}$, Schönaich, Germany) with a peak torque approximatively 10 times smaller than the larger motor. Each was driven by analog current/voltage amplifiers (model LCAM, Quanser, Markham, Ontario, Canada). Each shaft had a digital encoder (Mercury M1800, MicroE Systems, Bedford, MA, USA) giving 1,638,400 counts per turn.

The system seen in Fig. 3 was manufactured using a combination of conventional machining and rapid prototyping (ABS plastic), which was particularly effective for those parts which had to
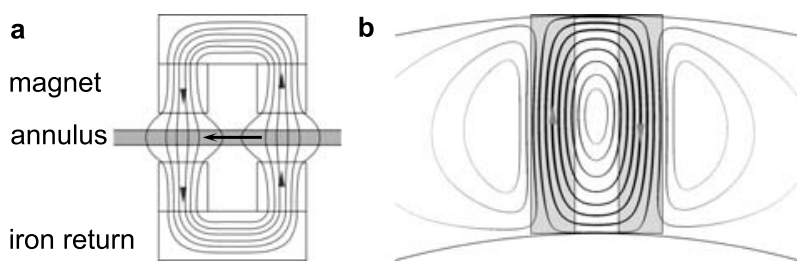

Figure 5: Results of magnetic simulation. a shows the magnetic field dispersion in the gap and $\mathbf{b}$ the predicted eddy currents in relation to the polar pieces (shadows shown in grey).

be lightweight. Some structural engineering was performed to ensure that all the parts had the required rigidity.

The magnetic circuits were optimized to reach the highest performance characteristics possible. According to reference [10], the basic characteristic of a viscous clutch is the ratio of its inertia to its viscous coefficient, $J / b$, which has units of time and expresses a time constant (for angular as well as for linear varieties). A viscous clutch has more parameters than a viscous brake since there are two rotors, the permanent magnet inductor and the driven disk which plays the role of an armature, thus, the number of factors to trade is larger. Nevertheless some design characteristics are similar. Among them is the need to maximize viscous drag. Viscous drag is a function of electrical conductivity and thickness of the armature, of the magnetic flux, of the geometry of the polar pieces, and of the air gap when accounting for losses and the skin effect. In reference [10] it was found experimentally to be advantageous to increase the aspect ratio of the polar pieces in the orthogonal direction of movement and to diminish the thickness of the armature to enhance the device time constant.

From the guidelines stated in reference [10], the magnet and armature arrangement depicted in Fig. 5a was selected. The viscous clutch comprises neodymium magnets with a remanent field of $1.37 \mathrm{~T}$ and an aluminum annulus, $0.6 \mathrm{~mm}$ thick, $10 \mathrm{~mm}$ wide, and $90 \mathrm{~mm}$ in outer diameter that is held by a light-weight spoked wheel as seen in Figs. 3 and 1. This wheel is connected to the small motor shaft. Much room is left for improvement in future designs.

Figures $5 \mathrm{a}$ and $5 \mathrm{~b}$ were obtained by simulating the magnetic behavior of the geometry using COMSOL 3.5 (COMSOL AB, Stockholm, Sweden). The mesh resolution was $0.3 \mathrm{~mm}$ for the regions of the armature shadowed by the magnets and coarser elsewhere. An important criterium for choosing the mesh resolution was to mesh the boundary of the annulus with element sizes sufficiently small to account for the skin effect. In the present geometry the skin depth was $5.3 \mathrm{~mm}$ for a speed of $45 \mathrm{rad} \cdot \mathrm{s}^{-1}$, hence invading the whole armature at higher speeds. Typically convergence was achieved in 15 steps for a relative precision of $10^{-6}$. Fig. 5a shows a cutaway view of the two magnetic circuits used in the two inductors seen on Fig 1. Fig. 5b shows how induced current would flow in the inductor as predicted by the simulation when there is relative movement. As can be seen, eddy currents creates three magnetic dipoles that oppose movement by interacting with the magnetic poles of the magnets.

\section{EXPERIMENTAL RESULTS}

\subsection{Identification}

The distal stage operates in open-loop and its dynamics is uncompensated. It is of paramount importance to obtain accurate estimates of its parameters. They were measured as follows. First, the distal stage's torque constant was measured keeping in mind that its electrical dynamics is governed by $u_{2}=k_{2} \dot{\theta}_{2}+R_{2} i_{2}+L_{2} \dot{i}_{2}$ when connected to a voltage amplifier, where $k_{2}$ is the sought torque constant, $u_{2}$ is the terminal voltage, $R_{2}$ is the winding resistance, $L_{2}$ is the inductance, and $i_{2}$ is the current. In steady 

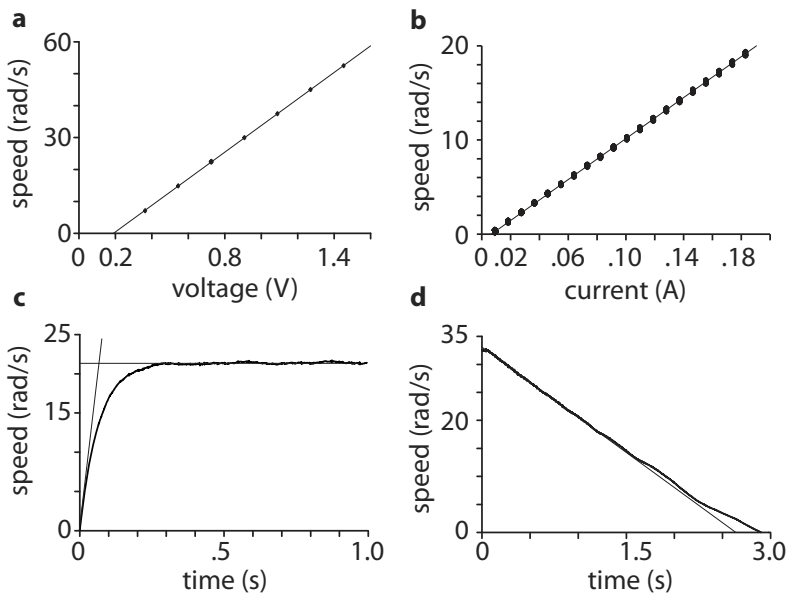

Figure 6: Raw data from measurement of the distal stage. Thin lines show the results of the regressions. Panel a shows how the torque constant was found; $\mathbf{b}$ shows the coefficient of the viscous coupler was found; c shows how the moment of inertia was found; and $\mathbf{d}$ shows how the friction torques was found.

state, when the coupler is disconnected, the mechanical response is $\dot{\theta}_{2}=1 / k_{2} u_{2}-R_{2} / k_{2} i_{2 \text { fric }}$, where $i_{2 \text { fric }}$ is the current needed to overcome friction. By varying the speed, $k_{2}$ could be backed out of the measurements by linear regression, see Fig. 6a. Next, the viscous coupler was reconnected and the proximal stage clamped. The new dynamics, in the steady state, was $\dot{\theta}_{2}=k_{2} i_{2} / b-\tau_{2 \text { fric }} / b$ when the amplifier was switched to current mode. Similarly $b$ could be estimated by linear regression, see Fig. $6 \mathrm{~b}$. To find the moment of inertia, a current step was given to the motor with the same conditions as before and the moment of inertia was deduced from the final angular velocity, $J_{2}=b \dot{\theta}_{2} / p$, where $p$ is the initial slope, see Fig. 6c. Finally, to find the friction torque, observe that if the handle is given an initial velocity, because of dry friction, the speed will decay at a constant rate, $\dot{\theta}_{2}=\dot{\theta}_{2}(0)-\tau_{2 \text { fric }} t / J_{2}$. From this response, the friction torque could be measured by linear regression, see Fig. 6d. The results are collected in Table 2.

\subsection{Time-Domain Performance}

The system was tested by holding the manipulandum with a normal grip and demanding a step of torque with the feedback control turned on $\left(K_{p}=0.11, T_{i}=0.03 \mathrm{~s}\right)$. The saturation levels were set to $8.5 \mathrm{mN} \cdot \mathrm{m}$ for the small motor and $45 \mathrm{mN} \cdot \mathrm{m}$ for the large one. The sampling rate was $5 \mathrm{kHz}$ and the data logging rate was $500 \mathrm{~Hz}$. Fig. 7 shows the results of demanding an up-step and a down-step of amplitude $10 \mathrm{mN} \cdot \mathrm{m}$ each at time $t=0$. The output torque is the sum of the clutch torque and of the small-motor torque, $\tau_{\text {out }}=\tau_{\text {clutch }}+\tau_{2}$. In Fig. $7 \mathrm{a}$, it can be seen how the large motor, as can be expected, received a large command spike. Since it is working against the viscous coupler, this condition results in a smoothly rising torque over a $40 \mathrm{~ms}$ duration. The missing torque is taken up by the small motor resulting in a sharp output torque tracking the demand precisely. The imperfection is a rounded corner near the set point due to the small motor saturation. In the second example, Fig. $7 b$, friction contributes to damp the large motor which results in a perfectly sharp, unconditionally stable transient lasting less than $2 \mathrm{~ms}$. In general, the mechanical time constant of the distal stage depends on the input step amplitude. For an up-step under the saturation level of the small motor, it is equal to the electrical time constant of the small motor, which is almost instantaneous.
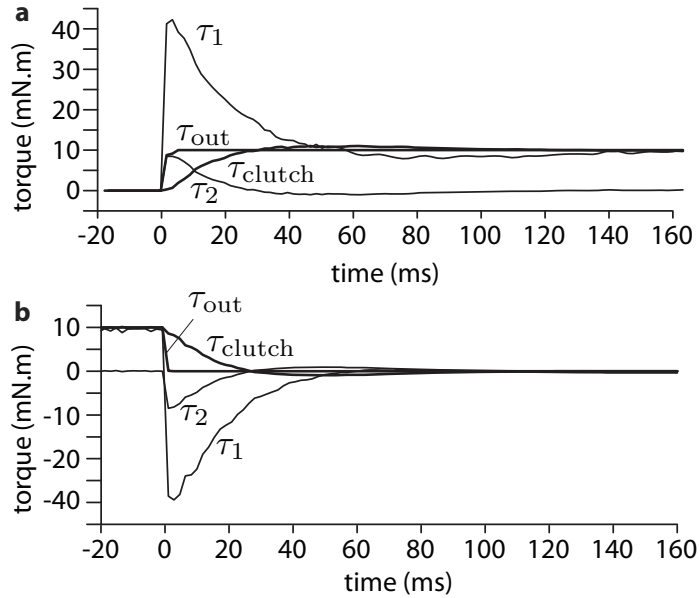

Figure 7: Time domain performance.

Table 2: Measured system parameters.

\begin{tabular}{lcc} 
Quantity & Unit & Value \\
\hline$k_{2}$ & $\mathrm{~N} \cdot \mathrm{m} \cdot \mathrm{A}^{-1}$ & $2410^{-3}$ \\
$b$ & $\mathrm{~N} \cdot \mathrm{m} \cdot \mathrm{s} \cdot \mathrm{rad}^{-1}$ & $2210^{-5}$ \\
$J_{2}$ & $\mathrm{~kg} \cdot \mathrm{m}^{2}$ & $1510^{-6}$ \\
$\tau_{2 \text { fric }}$ & $\mathrm{N} \cdot \mathrm{m}$ & $1910^{-5}$ \\
$\tau_{1 \max }$ & $\mathrm{N} \cdot \mathrm{m}$ & $4510^{-3}$
\end{tabular}

\subsection{Discussion}

While the advantages over a single motor design are not immediatetly apparent in the presently described proof-of-concept prototype, we anticipate two key advantages of the dual-motor design to be leveraged in future prototypes. Firstly, the effective inertia would always be determined by the inertia of the output moving parts, which become advantagous when high torques are needed and therefore when larger motors are used. In fact we expect this inertia to be always dominated by the handle but not by the motor. Our initial design does not achieve this because the large motor is too small and the disk too large. Similarly, the friction that the user experiences would always be limited to the friction due to the small motor instead of that of the large motor.

As can be seen from inspection of Table 1 and Table 2, the resulting system is not very far from meeting all the performance figures, which is an encouraging result given that the off-shelf parts were not specifically specified. Where the device is the weakest is in the maximum torque it can deliver. There is also too much friction by a factor 5 .

None of these limitations present obstacles that could not be surmounted with extra care in the design of a second prototype which is under construction at the time of writing. The friction due to the brushes of small DC motors is typically about $10^{-4} \mathrm{~N}$. For achieving smaller friction, brushless technologies such as galvanometric motors or limited angle torque motors could be considered. We expect the next prototype to be able to meet the requirements set out in Table 1 and hence to be a haptic device which for all practical purposes is completely "transparent" to a human user as all parasitic forces will be reduced to sub-threshold levels, while being able to produce high torques within an extended bandwidth at low signal distortion levels. 


\section{Conclusion}

We set out to design a single-axis haptic device capable of producing controllable forces that match the human motor and sensory performance, hence achieving perfect transparency. A dual stage design was selected and the preferred coupling method was a viscous clutch based on the principle of eddy currents. The objective of the closed loop control is to prevent the user to experienced the dynamics of the large proximal stage. The viscous coupler makes this objective somewhat easy to achieve since the system to be controlled reduces to what is essentially a first-order system. The distal stage supplies the missing transient in open-loop, yielding a system that is expected to be intrinsically robust and unconditionally stable without compromising performance.

Future work includes further optimizion of the design. Additional engineering effort could yield integrated designs that would have a form factor similar to that of standard motors with the two rotors coupled by an eddy current inductor inside a common package. Such dual-stage motors could then be conveniently integrated into intrinsically-safe, high-performance robotic and haptic systems intended to interact with humans.

More generally, we believe that we have first proposed a macroscale electromechanical system which can rightly be considered as a source of force with extremely low internal impedance $\left(<10^{3} \mathrm{~N}\right)$, negligible undesirable dynamics, a wide dynamic range (1:10.000), and a bandwidth as wide as the small motor. As such, its applications should far exceed those of haptic devices themselves.

\section{ACKNOWLEDGEMENTS}

The authors wish to thank Andrew Gosline for advice on magnetic design of viscous couplers and Bruno Sauvet for help with prototype manufacturing. This work was supported by the ANR (Agence Nationale de la Recherche, France), project PACMAN "perception haptique des échelles micro et nanoscopiques".

\section{REFERENCES}

[1] A. Bicchi, M. Peshkin, and J. E. Colgate. Safety for physical humanrobot interaction. In Springer Handbook of Robotics, chapter 57, pages 1335-1348. Springer-Verlag, 2008.

[2] B. Boulet. Modeling and control of a robotic joint with inparallel redundant actuators. Master's thesis, Deptartment of Electrical Engineering, McGill University, Montréal, Qc, Canada, 1990.

[3] G. Campion, A. H. Gosline, and V. Hayward. Passive viscous haptic textures. In Proceedings of the 16th Symposium on Haptic Interfaces For Virtual Environment And Teleoperator Systems, pages 379-380, 2008.

[4] M. Chew, G.-S. Hong, and W. Zhou. Series damper actuator: a novel force/torque control actuator. In Proceedings of the 4th IEEE/RAS International Conference on Humanoid Robots, pages 473-478, 2004.

[5] F. Conti, O. Khatib, and C. Baur. A hybrid actuation approach for haptic devices. In Proceedings of the Second Joint Eurohaptics Conference and Symposium on Haptic Interfaces for Virtual Environment and Teleoperator Systems, World Haptics 2007, pages 367-372, 2007.

[6] W. H. Doherty. A new high efficiency power amplifier for modulated waves. Proceedings of the IRE, 24(9):1163-1182, 1936.

[7] J. Dosher and B. Hannaford. Human interaction with small haptic effects. Presence: Teleoperators and Virtual Environments, 14(3):329$344,2005$.

[8] C. English and D. Russell. Implementation of variable joint stiffness through antagonist actuation using rolamite springs. Mechanisms and Machine Theory, 34(1):27-40, 1999.

[9] P. M. Fitts. The information capacity of the human motor system in controlling the amplitude of movement. Journal of Experimental Psychology, 47(6):381-391, 1954.

[10] A. H. Gosline and V. Hayward. Eddy current brakes for haptic interfaces: Design, identification, and control. IEEE/ASME Transactions on Mechatronics, 13(6):669-677, 2008.

[11] V. Hayward and O. R. Astley. Performance measures for haptic interfaces. In G. Giralt and G. Hirzinger, editors, Robotics Research:
The 7th International Symposium, pages 195-207, Heidelberg, 1996. Springer-Verlag.

[12] R. L. Hollis, S. E. Salcudean, and A. P. Allan. A six-degree-offreedom magnetically levitated variable compliance fine-motion wrist: Design, modeling, and control. IEEE Transactions on Robotics and Automation, 7(3):320-332, 1991.

[13] R. S. Johansson and R. H. Lamotte. Tactile detection thresholds for a single asperity on an otherwise smooth surface. Somatosensory and Motor Research, 1(1):21-31, 1983.

[14] O. Khatib. Reduced effective inertia in macro-/mini-manipulator systems. In H. Miura and S. Arimoto, editors, Robotics Research 5, pages 279-284. MIT Press, 1990.

[15] M. Lauria, M.-A. Legault, M.-A. Lavoie, and F. Michaud. Differential elastic actuator for robotic interaction tasks. In Proceedings of IEEE International Conference on Robotics and Automation, pages 36063611, 2008.

[16] J. Miles. Applications and limitations of mechanical-electrical analogies. Journal of the Acoustical Society of America, 14(3):183-192, 1943.

[17] J. B. Morrell and J. K. Salisbury. In pursuit of dynamic range: Using parallel coupled actuators to overcome hardware limitations. In Proceedings of the 4th International Symposium on Experimental Robotics IV, volume 223 of Lecture Notes in Control and Information Sciences, pages 263-273. Springer-Verlag, 1995.

[18] M. A. Pastor, B. L. Day, E. Macaluso, K. J. Friston, and R. S. J. Frackowiak. The functional neuroanatomy of temporal discrimination. The Journal of Neuroscience, 24(10):2585-2591, 2004.

[19] T. C. Pataky, M. L. Latash, and V. M. Zatsiorsky. Viscoelastic response of the finger pad to incremental tangential displacements. Journal of Biomechanics, 38(7):1441-1449, 2005.

[20] G. A. Pratt and M. M. Williamson. Series elastic actuators. In Proceedings of the IEEE/RSJ International Conference on Intelligent Robots and Systems, pages 399-406, 1995.

[21] S. E. Salcudean and C. An. On the control of redundant coarse-fine manipulators. In Proceedings of the IEEE International Conference on Robotics and Automation, pages 1834-1840, 1989.

[22] A. Sharon and D. E. Hardt. Enhancement of robot accuracy using endpoint feedback and a macro-micro manipulator system. In Proceedings American Control Conference, pages 1836-1842, 1984.

[23] A. Sharon, N. Hogan, and D. E. Hardt. High bandwidth force regulation and inertia reduction using a macro/micro manipulator system. In Proceedings of the IEEE International Conference on Robotics and Automation, pages 126-132, 1988.

[24] M. A. Srinivasan, J. M. Whitehouse, and R. H. LaMotte. Tactile detection of slip: surface microgeometry and peripheral neural codes. Journal of Neurophysiology, 63(6):1323-1332, 1990.

[25] L. Stocco and S. E. Salcudean. A coarse-fine approach to forcereflecting hand controller design. In Proceedings of the IEEE International Conference on Robotics and Automation, pages 404-410, 1996.

[26] S. Stramigioli and V. Duindam. Variable spatial springs for robot control applications. In Proceedings of the IEEE/RSJ International Conference on Intelligent Robots and Systems, pages 1906-1911, 2001.

[27] S. Sugano, S. Tsuto, and I. Kato. Force control of the robot finger joint equipped with mechanical compliance adjuster. In Proceedings of the IEEE/RSJ International Conference on Intelligent Robots and Systems, pages 2005-2013, 1992.

[28] R. T. Verrillo. Effect of contactor area on the vibrotactile threshold. Journal of the Acoustical Society of America, 35(12):1962-1966, 1963.

[29] J. P. Walker. Current dumping audio amplifier. Wireless World, 81:560-562, December 1975.

[30] S. A. Wall and W. Harwin. A high bandwidth interface for haptic human computer interaction. Mechatronics, 11(4):371-387, 2001.

[31] H. E. Wheat, L. M. Salo, and A. W. Goodwin. Human ability to scale and discriminate forces typical of those occurring during grasp and manipulation. The Journal of Neuroscience, 24(13):3394-3401, 2004.

[32] M. Zinn, B. Roth, O. Khatib, and J. K. Salisbury. A new actuation approach for human friendly robot design. International Journal of Robotics Research, 23(4):379-398, 2004. 